ISSN-i 1390-7042 | IssN-e 2602-8484

\title{
ESTUDIO CINÉTICO DE LA DESCOMPOSICIÓN TÉRMICA DEL POLIETILENO DE ALTA DENSIDAD (HDPE) POSCONSUMO A DIFERENTES TASAS DE CALENTAMIENTO
}

\section{Kinetic study of the thermal decomposition of high density polyethylene (HDPE) post consumption at different heating rates}

Paúl Palmay

Escuela Superior Politécnica de Chimborazo (Ecuador) Grupo de Investigación Ambiental y Desarrollo (GIADE) ORCID: https://orcid.org/0000-0002-9172-4113

\author{
Renee Jaramillo \\ Escuela Superior Politécnica de Chimborazo (Ecuador) \\ Grupo de Investigación Ambiental y Desarrollo (GIADE) \\ ORCID: https://orcid.org/0000-0002-5455-2914
}

Carlos Medina

Escuela Superior Politécnica de Chimborazo (Ecuador)

Grupo de Investigación Ambiental y Desarrollo (GIADE)

ORCID: https://orcid.org/0000-0003-4916-7242

Recibido: septiembre de 2020 Aprobado: mayo de 2021 ARTÍCULO ORIGINAL

DoI: $10.29166 /$ revfig.v11i1.3132
PALABRAS ClAVE HDPe, cinética, degradación, descomposición térmica.

KEYWORDS HDPE, kinetics, degradation, thermal decomposition.

\section{RESUMEN}

El presente estudio tiene como objetivo determinar la cinética química de la reacción de degradación térmica del polietileno de alta densidad (HDPE) posconsumo mediante análisis termogravimétrico (TGA) a tasas de calentamiento de 5,10 y $15^{\circ} \mathrm{C} \mathrm{min}^{-1}$, usando atmósfera de nitrógeno a un flujo de $20 \mathrm{~mL} \mathrm{~min}^{-1}$, en condiciones dinámicas desde 25 a $900^{\circ} \mathrm{C}$. A partir de ello se obtuvieron los datos de degradación (masa vs. tiempo) y su primera derivada, que a su vez fueron aplicados en tres modelos cinéticos para determinar la energía de activación: Friedman (FR), Kissinger-Akahira-Sunose (KAS) y Flynn-Wall-Ozawa (FWo), tomando el modelo de reacción conocido como Esfera de Contracción (R2) característica para este tipo de plásticos. Se deduce cada uno de los modelos para el tratamiento de termoplásticos, estableciendo que el modelo que describe mejor la cinética de degradación para HDPE es el de Friedman, obteniendo valores de energía de activación de 281, 248 y $232 \mathrm{~kJ} \mathrm{kmol}^{-1}$ para las tasas de 5,10 y $15^{\circ} \mathrm{C} \mathrm{min}^{-1}$, respectivamente.

\section{ABSTRACT}

The thermal degradation of the post-consumer high-density polyethylene (HDPE) to determine the chemical kinetics is the objective of this research. It was developed through thermogravimetric analysis (TGA) at 5,10 and $15^{\circ} \mathrm{C}$ $\mathrm{min}^{-1}$ heating rates, under nitrogen atmosphere at $20 \mathrm{~mL} \mathrm{~min}^{-1}$ and dynamic conditions from 25 to $900^{\circ} \mathrm{C}$. The degradation process data (mass vs time) and the first derivative were obtained from that data and were applied in three isoconversional kinetic models to determine the activation energy: Friedman (FR), Kissinger-Akahira-Sunose (KAS) and Flynn-Wall-Ozawa (FWO), in addition to the reaction model known as Sphere of Contraction (R2). All the described models fit well for the thermoplastics treatment, however, the models that best describe the degradation kinetics for HDPE are the KAS and FWO models, in these models the activation energy were 281,248 and $232 \mathrm{~kJ} \mathrm{kmol}^{-1}$ for 5,10 and $15^{\circ} \mathrm{C} \mathrm{m^{-1 }}$ heating rates respectively. 


\section{INTRODUCCIÓN}

Los plásticos se producen en una variedad de formas, pesos y con diferentes niveles de duración aprovechando sus propiedades, por lo que tienen muchas aplicaciones para el uso doméstico y en las diferentes industrias. Como consecuencia se generan grandes cantidades de estos residuos, de todo el plástico residual generado en el mundo, se estima que hasta un $70 \%$ de ellos son vertidos en rellenos sanitarios y el resto es incinerado (Khedri y Elyasi, 2016; Lovás et al., 2017). La disposición en vertederos no es la mejor opción, ya que los plásticos al no ser biodegradables permanecen cientos de años en el ambiente.

Se considera que los residuos sólidos urbanos (RSU) son la principal fuente de desechos plásticos (Xu et al., 2018) y el polietileno de alta densidad (HDPE) representa aproximadamente el $22,7 \%$ en peso de los plásticos en estos residuos (Thomas et al., 2020). La reutilización del HDPE tiene una creciente importancia en los últimos años, debido a la mayor demanda de recursos reciclables y protección del medioambiente. Convertir el plástico de desecho en hidrocarburos comercializables es una forma prometedora de obtener réditos económicos, además de los beneficios ecológicos. Los polímeros termoplásticos, y específicamente el HDPE, se considera como una fuente potencial de combustible, debido a su alto poder calorífico (43,3-46,5 kJ. $\left.\mathrm{kg}^{-1}\right)$; pudiendo descomponerse térmicamente (Al-Salem et al., 2017) en una serie de sustancias químicas valiosas, ofreciendo alternativas nuevas a la combustión tradicional (Al-Salem, 2019).

Hoy en día, la mayor parte del esfuerzo de investigación se enfoca en el reciclaje con técnicas avanzadas como la pirólisis (Khedri y Elyasi, 2016) (Thomas et al., 2020; Al-Salem et al., 2017) y la degradación catalítica mediante uso de zeolitas que ayuden a la formación de compuestos con características similares a las de combustibles convencionales (Arandes et al., 1997; Xue et al., 2017), dado que, la combustión como método de reciclaje genera graves problemas ambientales (Heidbreder et al., 2019). La descomposición térmica de residuos plásticos por pirólisis tiene ciertas ventajas sobre otras formas de reciclaje como, por ejemplo, la notable reducción de productos gaseosos (Arandes et al., 1997; Xue et al., 2017), así pues, aprovechando las ventajas que presenta el polietileno, se transforma los residuos del HDPE mediante degradación térmica en atmósferas inertes (pirólisis) (Anuar Sharuddin et al., 2016), donde las diferentes condiciones de operación producen varios productos de interés y su mayor ventaja es que estos productos son principalmente líquidos, por lo que pueden usarse directamente como combustible o transformarse en productos químicos valiosos.

La evaluación de los datos del análisis termogravimétrico (TGA) para la pirólisis de HDPE ha atraído la atención de varios investigadores (Das y Tiwari, 2017; Anuar Sharuddin et al., 2016; Charde et al., 2018) en los cuales se han estudiado la energía de activación de la pirólisis de HDPE para ser calculada a partir de los datos obtenidos del TGA a diferentes velocidades de calentamiento (Al-Salem et al., 2017; Thomas et al., 2020; Arandes et al., 1997). Estos y otros estudios han utilizado los modelos isoconversionales (Gutiérrez y Palza, 2015; Friedman, Kissinger-Akahira-Sunose, Flynn-Wall-Ozawa) para determinar la energía de activación, modelos con los que se ha demostrado que la pirólisis de HDPE es un proceso de un solo paso con una energía de activación de 238-247 kJ mol ${ }^{-1}$ (Aboulkas et al., 2010), 196,3-232,2 kJ mol-1 (Al-Salem y Lettieri, 2010), y 248,7 kJ mol ${ }^{-1}$ (Ceamanos et al., 2016).

En este trabajo se investigó la cinética química de la reacción de degradación térmica del HDPE haciendo uso de análisis termogravimétrico (TGA) a tasas de calentamiento de 5,10 y $15^{\circ} \mathrm{C}$ min $^{-1}$ bajo atmósfera de nitrógeno a un flujo de $20 \mathrm{ml} . \mathrm{min}^{-1} \mathrm{y}$ en condiciones dinámicas desde 25 a $900{ }^{\circ} \mathrm{C}$. La caracterización del HDPE se realizó por espectroscopia infrarroja por transformada de Fourier (FTIR). Se determinaron los parámetros cinéticos de la reacción de descomposición de residuos de HDPE a través de los tres modelos cinéticos antes mencionados, junto con el modelo de reacción conocido como Esfera de Contracción (R2), modelos que se ajustan al tratamiento de termoplásticos.

\section{MATERIALES Y MÉTODOS}

\section{CARACTERIZACIÓN DEL HDPE}

La caracterización del HDPE se la efectuó mediante espectroscopia infrarroja por transformada de Fourier (FTIR) para lo cual se realizó un muestreo completamente aleatorio de una muestra de $1 \mathrm{~kg}$ de plástico reciclado, el mismo que fue lavado con una solución de sosa cáustica al 1\%, secado y pulverizado para su posterior caracterización.

\section{ANÁLISIS TERMOGRAVIMÉTRICO}

Se realizaron análisis dinámicos en el equipo TGA 1 STAR System (Mettler Toledo), a tres tasas de calentamiento 5,10 y $15{ }^{\circ} \mathrm{C} \mathrm{min}^{-1}$, con atmósfera inerte mediante inyección de nitrógeno, el cual fue inyectado a un flujo $20 \mathrm{ml} \mathrm{min}^{-1}$. Con estos datos se determinó la pérdida de masa del HDPE frente al tiempo. 


\section{MODELO DE REACCIÓN}

Para un mejor ajuste de los datos del análisis termogravimétrico se usa el modelo del mecanismo de reacción estimado en reacciones de estado sólido de polietileno de alta densidad, que se denomina modelo de esfera de contracción: reacción de límite de fase bidimensional R2, el mismo que plantea que la función $f()$ es igual a $2(1-\alpha)^{1 / 2}$ mientras que la función $g(\alpha)$ es igual a 1-(1- $\alpha)^{1 / 2}$. Recomendado por Alboulkas et al. (2010), Das y Tiwari (2017) y Gao et al. (2003), para el análisis de HDPE, ya que es el modelo que mejor se ajusta para este material, de esta manera, el modelo de contracción geométrica supone que la velocidad de degradación inicia en la superficie, y a su vez está controlada por el avance de la reacción. Este comportamiento se debe a la facilidad de desintegración de las cadenas poliméricas gracias a la estructura lineal del HDPE.

\section{CINÉTICA DE LA DEGRADACIÓN TÉRMICA DE HDPE RECICLADO}

Para la degradación de polímeros, se usaron tres modelos isoconversionales en los que se asume que las tasas de conversión son proporcionales a la concentración del material reaccionado (Aboulkas et al., 2010). La velocidad de reacción se puede expresar mediante la siguiente ecuación:

$$
\frac{d \alpha}{d t}=\beta \frac{d \alpha}{d T}=k(T) f(\alpha) \quad E c .1
$$

Donde, $\beta$ es la tasa de calentamiento, $\alpha$ es la conversión, $\mathrm{f}(\alpha)$ es la función de conversión, $\mathrm{k}(\mathrm{T})$ función de temperatura.

El coeficiente de conversión permite cuantificar la pérdida de masa o su degradación:

$$
\alpha=\frac{m_{i}-m}{m_{i}-m_{f}} \quad \text { Ec. } 2
$$

Donde, $m_{i}$ es la masa inicial, $m$ es la masa a un tiempo determinado de degradación, $m_{f}$ es la masa final o residual.

$\mathrm{k}(\mathrm{T})$, la dependencia de la temperatura de la tasa de pérdida de peso, se determina con la ecuación de Arrhenius (Aboulkas et al., 2010), que es el fundamento de los modelos

$$
k(T)=A e^{\left(-\frac{E}{R T}\right)} \quad \text { Ec. } 3
$$

Donde, E es la energía de activación $\left(\mathrm{kJ} \cdot \mathrm{mol}^{-1}\right)$, A es el factor pre-exponencial $\left(\mathrm{s}^{-1}\right)$, $\mathrm{R}$ es la constante del gas $\left(0,008314 \mathrm{~kJ} \cdot \mathrm{mol}^{-1} \cdot \mathrm{K}^{-1}\right)$.

Combinando las ecuaciones (1) y (3), la velocidad de reacción se puede escribir:

$\beta \frac{d \alpha}{d T}=A e^{\left(-\frac{E}{R T}\right)} f(\alpha) \quad E c .4$

\section{MODELO CINÉTICO 1: MÉTODO DE FRIEDMAN (FR)}

Método isoconversional diferencial propuesto por Friedman, se basa directamente en la ecuación 4, cuyo logaritmo y aplicando el modelo de reacción R2 para $f(\alpha)($ Das y Tiwari, 2017), se tiene:

$\ln \left(\frac{d \alpha}{d t}\right)=\ln \left(\beta \frac{d \sigma}{d T}\right)=\ln (A)-\frac{E}{R T}+\ln \left(2(1-\alpha)^{1 / 2}\right) \quad E c .5$

\section{MODELO CINÉTICO 2: MÉTODO DE KISSIN- GER-AKAHIRA-SUNOSE (KAS)}

Método isoconversional integral, que se obtiene mediante otro ajuste de la integral de la ecuación 4; dicha ecuación estándar se puede representar de la siguiente forma:

$$
\frac{d \alpha}{f(\alpha)}=\frac{A}{\beta} e^{\left(-\frac{E}{R T}\right)} d(T) \quad \text { Ec. } 6
$$

Considerando que:

$$
\frac{d \alpha}{f(\alpha)}=g(\alpha) \quad \text { Ec. } 7
$$

Se integra con las condiciones iniciales de $\alpha=0$ en $T$ $=\mathrm{T} 0$. La técnica asume que $\mathrm{A}, \mathrm{f}(\alpha)$ y E son independiente de $\mathrm{T}$ mientras que $\mathrm{A}$ y $\mathrm{E}$ son independientes de $\alpha$, entonces, se tiene:

$$
g(\alpha)=\frac{A E}{\beta T}\left[\frac{e^{\left(-\frac{R}{R T}\right)}}{\frac{E}{R T}}-\int_{-\infty}^{E / R T} \frac{e^{\left(-\frac{R}{R T}\right)}}{\frac{E}{R T}} d(T)\right]
$$

Se define la siguiente relación:

$$
P\left(\frac{E}{R T}\right)=\frac{e^{\left(-\frac{E}{R T}\right)}}{\frac{E}{R T}}-\int_{-\infty}^{E / R T} \frac{e^{\left(-\frac{E}{R T}\right)}}{\frac{E}{R T}} d(T) \quad \text { Ec. } 9
$$


Entonces, se reemplaza $\mathrm{P}\left(\frac{E}{R T}\right)$, basándose en la aproximación de Coats-Redfern para dicho valor y aplicando logaritmo, tenemos:

$$
\ln (g(\alpha))=\ln \frac{A R}{E}+\ln \frac{T_{m}^{2}}{\beta}-\frac{E}{R T}
$$

Aplicando el modelo de reacción R2 para g ( $\alpha$ ), tene-

$$
\ln \frac{\left(1-(1-\alpha)^{1 / 2}\right)}{T_{m}{ }^{2}}=\ln \frac{A R}{E}-\ln \beta-\frac{E}{R T} \quad E c .11
$$

Se grafica $\frac{\left(1-(1-\alpha)^{1 / 2}\right)}{T_{m}{ }^{2}}$ vs. $1 / \mathrm{T}$; a partir de la pendiente se obtiene la energía de activación (E) y del punto de la ordenada se obtiene el factor pre exponencial (A).

\section{MODELO CINÉTICO 3: MÉTODO DE FLYNN-WALL -OZAWA FWO}

Se deriva del método isoconversional integral. La principal diferencia con el modelo de KAs es que FWO utiliza la aprnximarión de Doyle (Sakakibara et al., 1989) para : $\ln P\left(\frac{E}{R T}\right)$

$$
\ln P\left(\frac{E}{R T}\right)=-5.331-1.052 \frac{E}{R T}
$$

Relacionando las ecuaciones (10) y (12), y aplicando el modelo de reacción R2 para $\mathrm{g}(\alpha)$ se tiene:

$$
\ln \left(1-(1-\alpha)^{1 / 2}\right)=\ln \frac{A R}{E}-\ln \beta-5.331-1.052 \frac{E}{R T} \quad \text { Ec. } 13
$$

Se grafica $\left(1-(1-\alpha)^{1 / 2}\right)$ vs. 1/T; a partir de la pendiente se obtiene la energía de activación (E) y del punto de la ordenada se obtiene el factor pre-exponencial (A).

\section{RESULTADOS}

\section{CARACTERIZACIÓN DEL HDPE}

En la figura 1 se puede observar el espectro de la muestra de HDPE, resultado del análisis de una muestra aleatoria de un $1 \mathrm{~kg}$ de plástico triturado y lavado; proveniente de residuos plásticos urbanos recolectados durante 3 meses. De esta forma se pudo establecer los compuestos correspondientes a los valores medidos (ver Figura 1).

En el espectro FT-IR, el pico a $2913,91 \mathrm{~cm}^{-1}$ corresponde a la vibración de estiramiento del metil y metileno. A $2847,38 \mathrm{~cm}^{-1}$ se muestra una banda de vi- bración de estiramiento de grupos metoxi debido a la presencia de antioxidantes que se usan como aditivo en la obtención de productos a base de este plástico. La banda de 1471,42 $\mathrm{cm}^{-1}$ corresponde a la vibración de estiramiento de azotriasoles (presencia de absorbedores Uv) y alcanos. A $717,39 \mathrm{~cm}^{-1}$, la banda corresponde a la vibración de etilenos que se presentan por el tratamiento térmico, extrusión, al cual se somete el HDPE virgen. Los resultados obtenidos son muy similares a los presentados en el estudio de Krehula et al. (2014), con la diferencia de que, en la investigación de Krehula et al. (2014) no existe bandas de vibración correspondientes a los grupos metoxi y azotriazoles, pues estos autores utilizaron HDPE puro (Ceamanos et al., 2016; Gutiérrez y Palza, 2015).

\section{Análisis termogravimétrico}

En la figura 2 se muestra la variación de la masa con respecto a la temperatura para las tres tasas de calentamiento (TGA), mientras que en la figura 3 se observa la derivada de la curva de pérdida de masa respecto a la temperatura (DTG) mediante la cual se estableció la temperatura máxima de degradación, siendo estas $740,749,757 \mathrm{~K}$ para 10, 15 y $20^{\circ} \mathrm{C} / \mathrm{min}$ (ver Figuras 2 у 3$)$.

\section{Modelo cinético}

A continuación, se presenta la tabla 1, que muestra los resultados obtenidos de cada modelo para las diferentes tasas de calentamiento (ver Tabla 1 y Figura 4).

\section{DISCUSIÓN}

De acuerdo al termograma la reacción de degradación del polietileno se lleva a cabo en una sola etapa consiguiendo una pérdida máxima de masa a la temperatura de degradación indicada para cada tasa de calentamiento, lo que muestra un mecanismo de reacción igual en cada tasa (Singh, 2020); donde claramente la pendiente en los tres casos se mantiene, sin la presencia de fluctuaciones en la medida de la masa, marcando el rango para el mínimo y máximo porcentaje de pérdida. Rango que es utilizado para el planteamiento de los modelos. Este comportamiento hace notar la gran correlación de los datos con los modelos propuestos en las tres tasas. Teniendo, por tanto, para la tasa de calentamiento de $5^{\circ} \mathrm{C} \cdot \mathrm{min}^{-1}$ se estima una energía de activación de $266,78 \mathrm{~kJ} \cdot \mathrm{mol}^{-1}$, y un factor preexponencial de $8,11 \times 10^{16} \mathrm{~s}^{-1}$, en cambio para la tasa de $10^{\circ} \mathrm{C} . \mathrm{min}^{-1}$ una energía de activación de $247,745 \mathrm{~kJ} \mathrm{~mol}^{-1}$, y un factor preexponencial de $5,068 \times 10^{15} \mathrm{~s}^{-1} \mathrm{y}$, por último, para $15^{\circ} \mathrm{C} \cdot \mathrm{min}^{-1}$ una energía de activación de $231,842 \mathrm{~kJ}^{\mathrm{mol}}{ }^{-1}$, y un factor preexponencial de $4,154 \times 10^{14} \mathrm{~s}^{-1}$. El modelo que 


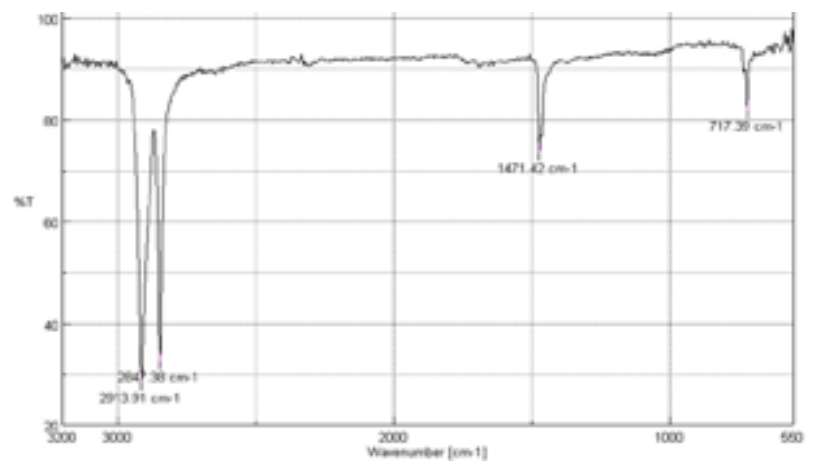

Figura 1. FT-IR del HDPE reciclado

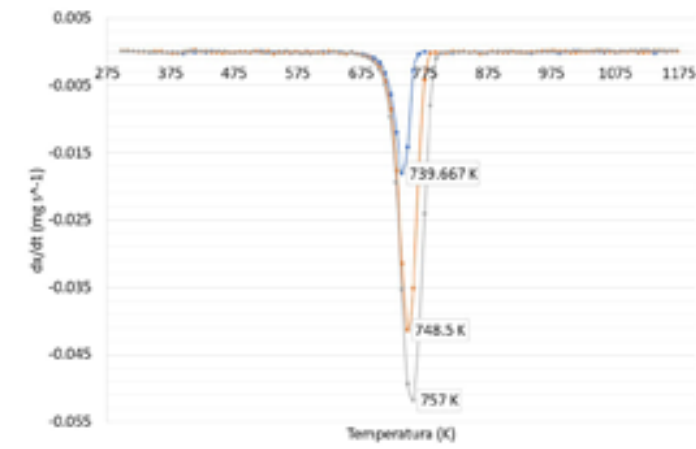

Figura 3. Derivada de la pérdida de masa de HDPE (DTG)

Figura 4. Comparación de los modelos FR, KAS y FWO frente al DTG experimental a diferentes tasas de calentamiento de 5,10 y $15^{\circ} \mathrm{C}$.min-1.

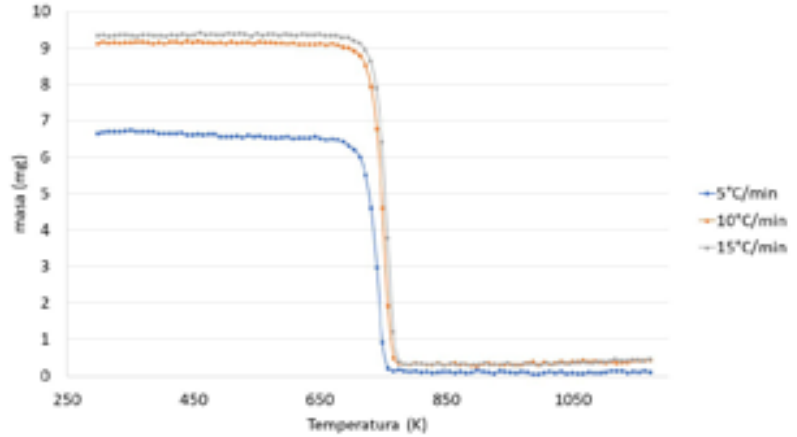

Figura 2. Perfil de la degradación térmica de HDPE reciclado (TGA)

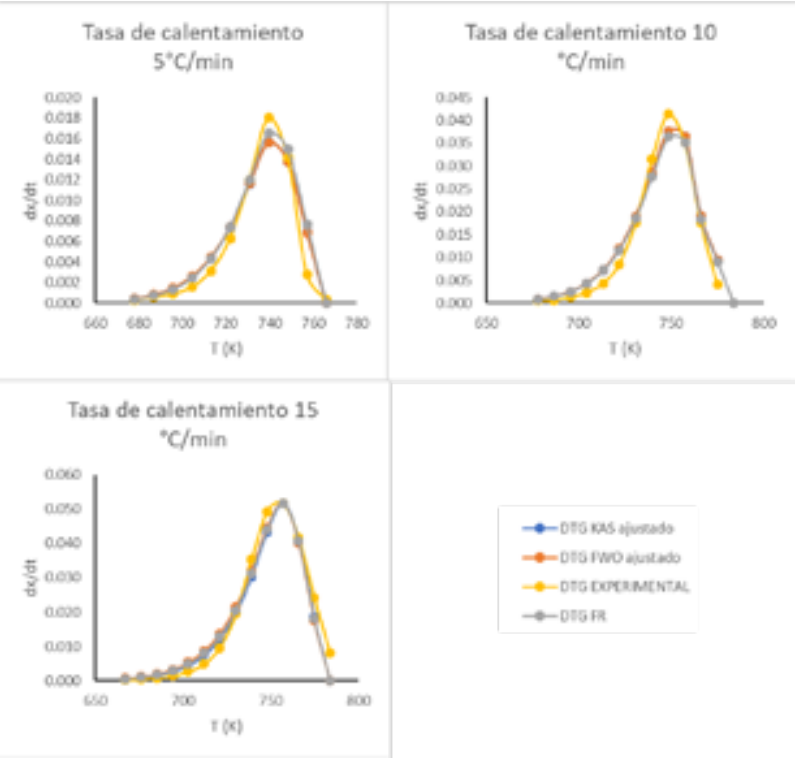

Tabla 1. Resultados de los modelos FR, KAS Y FWO

\begin{tabular}{|c|c|c|c|c|c|c|}
\hline \multirow{2}{*}{$\begin{array}{l}\stackrel{ }{1} \\
\frac{1}{2} \\
\frac{1}{2}\end{array}$} & \multirow{2}{*}{$\begin{array}{c}\text { B } \\
\left({ }^{\circ} \text { C.min }{ }^{-1}\right)\end{array}$} & \multicolumn{5}{|c|}{ RESULTADOS } \\
\hline & & $\operatorname{Tmax}(\mathbf{K})$ & Regresión Lineal & $\mathbf{R}^{2}$ & $\underset{\left(k J \cdot \mathrm{mol}^{-1}\right)}{\mathbf{E}}$ & $A\left(s^{-1}\right)$ \\
\hline \multirow{3}{*}{$\frac{x}{2}$} & 5 & 739.67 & $\ln \left(\frac{d \alpha}{d t}\right)=41.630-\frac{33826.68}{T}$ & 0.9944 & 281.235 & $8.994 \times 10^{17}$ \\
\hline & 10 & 748.50 & $\ln \left(\frac{d \alpha}{d t}\right)=36.496-\frac{29798.51}{T}$ & 0.9917 & 247.745 & $5.068 \times 10^{15}$ \\
\hline & 15 & 757.00 & $\ln \left(\frac{d \alpha}{d t}\right)=33.875-\frac{27885.71}{T}$ & 0.9977 & 231.842 & $4.154 \times 10^{14}$ \\
\hline \multirow{3}{*}{ 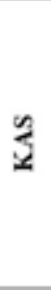 } & 5 & 739.67 & $\ln \frac{\left(1-(1-\alpha)^{1 / 2}\right)}{T_{m}^{2}}=31.380-\frac{32309.60}{T}$ & 0.9954 & 268.622 & $1.091 \times 10^{17}$ \\
\hline & 10 & 748.50 & $\ln \frac{\left(1-(1-\alpha)^{1 / 2}\right)}{T_{m}^{2}}=28.263-\frac{30204.44}{T}$ & 0.9881 & 251.120 & $9.003 \times 10^{15}$ \\
\hline & 15 & 757.00 & $\ln \frac{\left(1-(1-\alpha)^{1 / 2}\right)}{T_{m}^{2}}=25.990-\frac{28760.83}{T}$ & 0.9900 & 239.118 & $1.320 \times 10^{15}$ \\
\hline \multirow{3}{*}{ 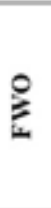 } & 5 & 739.67 & $\ln \left(1-(1-\alpha)^{1 / 2}\right)=46.465-\frac{33756.40}{T}$ & 0.9954 & 266.778 & $8.108 \times 10^{16}$ \\
\hline & 10 & 748.50 & $\ln \left(1-(1-\alpha)^{1 / 2}\right)=43.382-\frac{31674.97}{T}$ & 0.9881 & 250.329 & $7.929 \times 10^{15}$ \\
\hline & 15 & 757.00 & $\ln \left(1-(1-\alpha)^{1 / 2}\right)=38.734-\frac{28392.57}{T}$ & 0.9900 & 224.388 & $1.271 \times 10^{14}$ \\
\hline
\end{tabular}


más se ajusta es el de Friedman, sin embargo, no podemos pasar por alto que este modelo es simple en comparación con KAS y Fwo, ya que trabaja con menor cantidad de datos y variables que tienen una desviación mínima debido a su excelente correlación para múltiples datos de tasa de calentamiento. Los resultados son comparables con trabajos similares para la tasa de calentamiento de $5^{\circ} \mathrm{C} \cdot \mathrm{min}^{-1}$ con Das y Tiwari (2017), que obtuvieron valores de energía de activación de 241 hasta $258 \mathrm{~kJ} \cdot \mathrm{mol}^{-1}$, y el factor pre-experimental de $1,52 \times 10^{16}$ hasta $2,34 \times 10^{17}$. Así mismo, con Alboulkas et al. (2010) y Yang et al. (2001) los cuales presentan valores menores a los reportados, entendiéndose que una energía de activación más baja se refiere a que la degradación comienza en los enlaces más débiles de la cadena del polímero que se pueden atribuir a la presencia de aditivos que contienen enlaces nitrógeno-carbono o procesamientos previos para la obtención del plástico (Miandad et al., 2017).

Además, se puede observar como la tasa de calentamiento influye en el proceso de degradación, ya que, cuanto mayor es la tasa, mayor es la temperatura máxima de degradación y menores son tanto la energía de activación, como el factor preexponencial independiente del modelo utilizado (Li et al., 2021).

\section{CONCLUSIONES}

La degradación del polietileno reciclado sigue una reacción de degradación en una sola etapa mediante el mecanismo de reacción conocido como esfera de contracción. Proceso que por experimentación termogravimétrica se determinó la energía de activación y el factor preexponencial, siendo el modelo de Friedman el que más se ajusta a los datos obtenidos. Sin embargo, los datos solo presentan leves desviaciones a los estudios reportados por otros autores debido principalmente a la presencia de aditivos y el procesamiento del plástico puro, a diferencia del plástico posconsumo que se utilizó para la presente experimentación.

\section{REFERENCIAS}

Aboulkas, A., El harfi, K., y El Bouadili, A. 2010. Thermal degradation behaviors of polyethylene and polypropylene. Part I: Pyrolysis kinetics and mechanisms. Energy Conversion and Management, 51(7), 1363-1369. DOI: 10.1016/j.enconman.2009.12.017

Al-Salem, S. M. 2019. Thermal pyrolysis of high density polyethylene (HDPE) in a novel fixed bed reactor system for the production of high value gasoline range hydrocarbons (HC). Process Safety and Environmental Protection, 127(7), 171-179.

Al-Salem, S. M., Antelava, A., Constantinou, A., Manos, G. y Dutta, A. 2017. A review on thermal and catalytic pyrolysis of plastic solid waste (PSW). Journal of Environmental Management, 197(07), 177-98. DOI: 10.1016/j. jenvman.2017.03.084

Al-Salem, S. M. y Lettieri, P. 2010. Kinetic study of high density polyethylene (HDPE) pyrolysis. Chemical Engineering Research and Design, 88(12), 1599-1606. DOI: 10.1016/j.cherd.2010.03.012

Anuar Sharuddin, S. D., Abnisa, F., Wan Daud, W. M. A. y Aroua, M. K. 2016. A review on pyrolysis of plastic wastes. Energy Conversion and Management, 115(5), pp. 308-326. DoI: 10.1016/j.enconman.2016.02.037

Arandes, J. M., Abajo, I., López-Valerio, D., Fernández, I., Azkoiti, M. J., Olazar, M. 1997. Transformation of several plastic wastes into fuels by catalytic cracking. Industrial \& Engineering Chemistry Research, 36(11), 4523-4529.

Ceamanos, J., Mastral, J. F., Millera, A. y Aldea, M. E. 2002. Kinetics of pyrolysis of high density polyethylene. Comparison of isothermal and dynamic experiments. Journal of Analytical and Applied Pyrolysis, 65(2), 93-110. DoI: 10.1016/S0165-2370(01)00183-8

Charde, S. J., Sonawane, S. S., Sonawane, S. H. y Shimpi, N. G. 2018. Degradation kinetics of polycarbonate composites: kinetic parameters and artificial neural network. Chemical and Biochemical Engineering Quarterly, 32(2), 151-165. DoI: 10.15255/CABEQ.2017.1173

Das, P. y Tiwari, P. 2017. Thermal degradation kinetics of plastics and model selection. Thermochimica Acta, 654 (9), 191-202. DoI: 10.1016/j.tca.2017.06.001

Gao, Z., Amasaki, I. y Nakada, M. A. 2003. Thermogravimetric study on thermal degradation of polyethylene. Journal of Analytical and Applied Pyrolysis, 67(1), 1-9. DOI: 10.1016/S0165-2370(02)00010-4

Gutiérrez, O. y Palza, H. 2015. Effect of carbon nanotubes on thermal pyrolysis of high density polyethylene and polypropylene. Polymer Degradation and Stability, 120(10), 122-134. DOI: 10.1016/j.polymdegradstab.2015.06.014

Heidbreder, L. M., Bablok, I., Drews, S. y Menzel, C. 2019. Tackling the plastic problem: a review on perceptions, behaviors, and interventions. Science of the Total Environment, 668(6), 1077-1093. DOI: 10.1016/j.scitotenv.2019.02.437

Khedri, S., y Elyasi, S. 2016. Kinetic analysis for thermal cracking of HDPE: a new isoconversional approach, Polym Degrad Stab, 129(7), 306-318. DoI: 10.1016/j. polymdegradstab.2016.05.011

Krehula, L. K., Katancć, Z., Siročić, A. P. y Hrnjak-Murgić, Z. 2014. Weathering of high-density polyethylene-wood plastic composites. Journal of Wood Chemistry and Technology, 34(1), 39-54. DOI: 10.1080/02773813.2013.827209

Li, D., Lei, S., Wang, P., Zhong, L., Ma, W. y Chen, G. (2021). Study on the pyrolysis behaviors of mixed waste plastics. Renewable Energy, 173, 662-474. DoI: 10.1016/j. renene.2021.04.035

Lovás, P., Hudec, P., Jambor, B., Hájeková, E. y Horňáček, M. 2017. Catalytic cracking of heavy fractions from the pyrolysis of waste HDPE and PP. Fuel, 203(9), 244-252.

Miandad, R., Barakat, M. A., Aburiazaiza, A. S., Rehan, M., Ismail, I. M. I. y Nizami, A. S. (2017). Effect of plastic 
waste types on pyrolysis liquid oil. International Biodeterioration \& Biodegradation, 119(4), 239-252. DOI: 10.1016/j.ibiod.2016.09.017

Sakakibara, M., Okada, F., Horiuchi, M., Suzuki, K. 1989. Kinetic Analysis of thermogravimetric data. Nippon Kagaku Kaishi, 1989(10), 1729-1732.

Singh, R. K., Ruj, B., Sadhukhan, A. K. y Gupta, P. 2020. A TG-FTIR investigation on the co-pyrolysis of the waste HDPE, PP, PS and PET under high heating conditions. Journal of the Energy Institute, 93(3), 1020-1035. DoI: 10.1016/j.joei.2019.09.003

Thomas, P., Rumjit, N. P., Lai, C. W., Johan, M. R. B. y Saravanakumar, M. P. 2020. Polymer-recycling of bulk plastics. Encyclopedia of Renewable and Sustainable Materials, 2, 211-227.
Xu, F., Wang, B., Yang, D., Hao, J., Qiao, Y. y Tian, Y. (2018). Thermal degradation of typical plastics under high heating rate conditions by TG-FTIR: pyrolysis behaviors and kinetic analysis. Energy Conversion and Management, 171(4), 1106-1115.

Xue, Y., Johnston, P. y Bai, X. 2017. Effect of catalyst contact mode and gas atmosphere during catalytic pyrolysis of waste plastics. Energy Conversion and Management, 142(6), 441-451. DoI: 10.1016/j.enconman.2017.03.071

Yang, J., Miranda, R. y Roy, C. 2001. Using the DTG curve fitting method to determine the apparent kinetic parameters of thermal decomposition of polymers. Polymer Degradation and Stability, 73(3), 455-61. DoI: 10.1016/ S0141-3910(01)00129-X 